\title{
A Standardized Clinical Evaluation of Phenotypic Diversity in Diabetic Polyneuropathy
}

Joachim Scholza,b, James P. Rathmellc, William S. David ${ }^{d}$, David A. Chad ${ }^{d}$, Alithia C. Brodericke, Stephen G. Perros ${ }^{e}$, Naomi S. Shine, Jenna L. Wells ${ }^{d}$, John B. Davis ${ }^{\dagger}$, Charles J. DiMaggioa, Shuang Wang ${ }^{g}$, Simon N. Tate ${ }^{h}$

a Departments of Anesthesiology and b Pharmacology, Columbia University Medical Center, New York, NY, United States of America

c Department of Anesthesia, Perioperative and Pain Medicine, Brigham and Women's Hospital, Boston, MA, United States of America

d Department of Neurology, Massachusetts General Hospital, Boston, MA, United States of America

e Department of Anesthesia, Critical Care and Pain Medicine, Massachusetts General Hospital, Boston, MA, United States of America

f Selcia, Essex, United Kingdom

g Department of Biostatistics, Columbia University Mailman School of Public Health, New York, NY, United States of America

h Convergence Pharmaceuticals, Cambridge, United Kingdom

Corresponding Author Joachim Scholz, MD, Department of Anesthesiology, Columbia University Medical Center, 630 West 168 ${ }^{\text {th }}$ Street, P\&S Box 46, New York, NY 10032, United States of America. Phone 212-305-1274, fax 212-304-6539, e-mail js3932@cumc.columbia.edu

35 Pages, including pages containing figures and tables

247 Words in the Abstract

496 Words in the Introduction

1345 Words in the Discussion

8 Figures

1 Table

2 Files submitted as Supplemental Digital Content

Key words Diabetic polyneuropathy; neuropathic pain; pain assessment; phenotype 


\section{Abstract}

Diabetic polyneuropathy (DPN) is a major cause of neuropathic pain and a frequent target condition in analgesic treatment trials. Differences in the clinical symptoms and signs associated with DPN suggest distinct pathophysiological mechanisms underlying nerve damage and dysfunction that are likely to have therapeutic relevance. The aim of this study was to develop a tool for the bedside assessment of painful neuropathies such as DPN that captures the diversity of phenotypes. Sixty-one patients with type 2 diabetes (DM2) and painful neuropathy, 19 patients with painless DPN, 25 patients with DM2 but no clinical evidence of neuropathy and 20 healthy control subjects completed a structured interview (47 items) and a standardized physical examination (39 items). After analyzing critical features of pain and painless symptoms and examining the outcome of physical tests of sensory function, we determined the principal components of the phenotypic variance among patients. Increased sensitivity to mechanical or thermal stimuli and, to a lesser extent, the sensory quality of pain or paresthesia were the most discriminating elements of DPN phenotypes. Correlation patterns of symptoms and signs indicated the involvement of functionally distinct nerve fiber populations. We combined interview questions and physical tests identifying these differences in a shortened assessment protocol that we named Standardized Evaluation of Pain and Somatosensory Function (StEPS). StEPS generates a phenotypic profile of patients with neuropathy. Separate intensity ratings for spontaneous painful symptoms and pain evoked by standard stimuli support a detailed documentation of neuropathic pain and its response to analgesic treatment. 


\section{Introduction}

Patient stratification by phenotype is increasingly recognized as a promising approach to providing pain relief with greater precision and reducing outcome variation in analgesic treatment trials [5]. Diabetic polyneuropathy (DPN) is one of the leading causes of neuropathic pain and a frequent target condition in studies of neuroprotective or analgesic treatment. Up to $54 \%$ of patients with type 1 diabetes and $45 \%$ of patients with type 2 diabetes develop a clinical neuropathy [24,63]. DPN, which involves multiple somatic and autonomic nerves and produces symptoms and signs in a distal symmetrical distribution, is the most common manifestation [15]. The phenotype of DPN varies substantially. The majority of patients present with a variety of sensory deficits, whereas pain occurs in $16 \%$ to $21 \%$ of the patients $[1,19]$. Mild autonomic impairment can be detected in many patients with type 2 diabetes [39], although stringent criteria of autonomic neuropathy yield a lower prevalence of $22 \%$ [64]. Loss of motor function is less frequent [3]. The cause of this phenotypic diversity is unclear. Age, diabetes duration, poor glycemic control and vascular disease contribute to the overall risk of DPN but do not explain differences in symptoms and signs $[56,63]$. Nor do pathological findings in nerve or skin biopsies necessarily match the clinical phenotype. For example, deficits in temperature discrimination correlate inconsistently with the intraepidermal loss of temperature-sensitive C or Aס nerve fibers [21], and degenerative changes in nociceptive nerve fibers do not explain the manifestation of pain $[17,59]$.

Phenotypic variance in DPN is likely to result from a combination of genetic and pathophysiological factors $[9,15,60,62]$. A thorough standardized characterization of DPN's clinical features will be necessary to identify these factors and determine their potential correlation with treatment response [61]. Strategies for the assessment of the neuropathic phenotype should ideally provide comprehensive information about all relevant disorders of nerve function and be suitable for implementation in research trials as well as clinical practice. However, most questionnaires for DPN are deliberately short and designed to screen large study populations for key symptoms of the disease or physical impairment, not phenotypic variance $[14,23,26,53]$. Measures of nerve conduction, quantitative sensory testing (QST) and autonomic reflexes generate detailed information about nerve fiber function, 
but these investigations demand time, technical expertise and equipment that is typically only found in specialized neuromuscular centers $[30,37,38,55]$.

Here we have taken an alternative approach and developed a structured interview and standardized physical examination for the bedside evaluation of sensory function and pain in DPN. We prospectively compared patients with type 2 diabetes and healthy control subjects, and conducted a principal component analysis (PCA) to determine which combinations of clinical symptoms and signs distinguish DPN phenotypes. Previous studies of neuropathic pain included subjects with diverse neurological disorders, generating information that may not entirely apply to DPN $[11,42,50]$, especially painless variants of the disease $[6,12,29]$. To our knowledge, this is the first specific analysis of phenotypic variance in DPN that evaluates sensory loss and pain with a broad array of standardized clinical measures.

\section{Methods}

The study was performed at Massachusetts General Hospital in Boston, Massachusetts. Partners Human Research Committee, which serves as the institutional review board of Massachusetts General Hospital, approved the research (Protocol 2009P-001449). All patients gave written informed consent.

\subsection{Study participants}

We prospectively recruited 20 healthy control $(\mathrm{HC})$ subjects and 164 patients diagnosed with type 2 diabetes mellitus (DM2) according to the criteria established by the American Diabetes Association [2]. Patients gave authorization to confirm the diagnosis with their treating physician. All subjects were older than 18 years. Probable DPN as defined by the Toronto Diabetic Neuropathy Expert Group was independently diagnosed by two experienced neurologists (WSD, DAC) and a trained physician assistant (ACB). The diagnosis required a combination of $\geq 2$ neuropathic symptoms or signs including paresthesia, pain, sensory deficits in a distal symmetric distribution and unequivocally decreased or absent ankle reflexes [55]. Records of previous nerve conduction studies, skin or nerve biopsies were 
reviewed when available to confirm the diagnosis. Patients with DPN were classified into those with $(P)$ or without chronic pain (NoP) defined as persistent or recurrent pain for $\geq 3$ months [58]. If pain was present, its intensity had to be $\geq 4$ on a numerical rating scale (NRS) from 0 (no pain) to 10 (maximum imaginable pain) in the week before enrollment. This threshold was chosen to match the minimum pain intensity required for inclusion in many analgesic treatment trials [22,28]. All subjects with DPN-P fulfilled the criteria for definite neuropathic pain [57]. Subjects were excluded if they had another neurological or painful disorder, infection, severe medical or psychiatric illness, or if they were unable to give written informed consent. Previously prescribed analgesic treatment was allowed to continue.

\subsection{Diabetic neuropathy scores}

We determined the Diabetic Neuropathy Symptom (DNS) score, which documents unsteady gait, pain, paresthesia and numbness. The highest DNS score is 4 [43]. We also calculated the symptoms and sensory tests scores of the Toronto Clinical Neuropathy Scoring System (CNS). The symptoms score rates pain, numbness, tingling, weakness, ataxia and upper-limb symptoms. The sensory tests score is calculated based on the presence of abnormal responses to pinprick, temperature stimulation, light touch or vibration, or failure to recognize changes in the position of the big toe. The highest symptoms score is 6 , the highest sensory tests score is 5 [13].

\subsection{Assessment of sensory symptoms and signs}

The investigators evaluating symptoms and signs were blind to the diagnosis of diabetes or polyneuropathy. They were specifically trained in the evaluation protocol to ensure consistent test application and interpretation.

We designed the assessment of sensory symptoms and signs as an extension of the Standardized Evaluation of Pain (StEP), which we recently validated for the diagnosis of radicular back pain [50]. Symptoms were recorded in a structured interview (47 items). Questions addressed the physical location of pain, its temporal characteristics, sensory quality and dependence on external stimuli. If pain evoked by stimuli such as touch or cold was reported, we asked whether the pain was limited to 
the presence of the stimulus or persisted beyond the exposure (aftersensation). Subjects indicated the intensity of each aspect of their pain on an NRS from 0 to 10. Other interview items explored the presence of numbness or positive painless sensations (paresthesia) such as tingling or itch.

The physical examination (39 items) consisted of a standardized bedside evaluation of sensory signs and a brief assessment of peripheral autonomic functions. Quantitative tests of autonomic and motor functions are reported separately. We used two von Frey filaments (North Coast Medical) to examine the reaction to punctate mechanical stimulation at low $(2 \mathrm{~g})$ or high strength $(26 \mathrm{~g})$. To test the response to dynamic mechanical stimulation, we moved a soft brush (SENSELab 05; Somedic) at a constant speed of $3-5 \mathrm{~cm} / \mathrm{s}$ over the skin without changing direction. The response to pressure was tested with a rubber-tipped algometer (Wagner Instruments), applied for $10 \mathrm{~s}$ at steady low $\left(2 \mathrm{~kg} / \mathrm{cm}^{2}\right)$ or high force $\left(6 \mathrm{~kg} / \mathrm{cm}^{2}\right)$. For pinprick-evoked pain, we used a medium-size unbeveled pin. Warm $\left(40^{\circ} \mathrm{C}\right.$, $\left.45^{\circ} \mathrm{C}\right)$ and cold $\left(20^{\circ} \mathrm{C}, 5^{\circ} \mathrm{C}\right)$ stimuli were produced with a pen-shaped Peltier thermode of $1.25 \mathrm{~cm}$ diameter (NTE-2A; Physitemp Instruments). Each temperature was applied for $10 \mathrm{~s}$. We waited at least 1 min before changing the test temperature. The sense of vibration was assessed with a standard tuning fork $(128 \mathrm{~Hz})$ placed over the first metatarsophalangeal joint. Proprioception was evaluated by examining the sense of position and passive movement at the interphalangeal joint of the big toe. To test for temporal summation, the stronger of the two von Frey filaments $(26 \mathrm{~g})$ was applied repeatedly, at a rate of $1-2 \mathrm{~Hz}$ for 30 seconds. The test was considered positive if a painless response turned painful or evoked pain increased in its intensity during the stimulation. With the exception of temporal summation, each of the test stimuli was applied 4 times and the result considered positive when $\geq 3$ stimulations produced a consistent response. If pain was provoked, we asked the patient to rate the intensity of the pain using an NRS. All tests were performed on the dorsum of the foot and compared to responses on the dorsum of the hand or the forearm, if DPN involved the hands.

We also compared the sensitivity and specificity of pain detection with the Douleur Neuropathique en 4 Questions (DN4) screening tool for neuropathic pain [10]. DN4 items were integrated into the standardized evaluation of sensory symptoms and signs so that neither the patients nor the investigators 
were able to differentiate interview or examination items belonging to the DN4.

\subsection{Statistical analysis}

General subject characteristics were compared across groups in a one-way analysis of variance (ANOVA) followed by Tukey's test for multiple comparisons. Frequency distribution of sensory symptoms and signs was analyzed with Fisher's exact test. We performed a principal component analysis (PCA) to identify correlated symptoms and signs of DPN.evaluate and reduce the number and dimensions of phenotypic features, identify correlated symptoms and signs and determine the level of contribution of these variables to the phenotypic variance. The analysis allowed us to reduce the complexity of the phenotypic variance between subjects to the principal components of differences in their responses to interview questions and physical test outcomes. We included the phenotyping results of all patients with DM2 in this analysis, considering diabetes without polyneuropathy (DM2 w/o DPN) as reference condition. Items with high PCA component loadings were included in an abridged version of the assessment protocol. To determine the effectiveness of the full assessment and this reduced set of items in capturing sensory deficits and pain, we added the responses in each set of variables to a series of logistic regression models and compared the results against the independent neurological classification of the patients. Based on the separation between subgroups of patients, we generated receiver operating characteristic $(\mathrm{ROC})$ curves and calculated the area under the curves (AUCs) to assess accuracy. All analyses were performed using the Statistical Analysis System software program (SAS Institute). Venn diagrams were generated with the Venn Diagram Plotter (Pacific Northwest National Laboratory).

\section{Results}

\subsection{Subject characteristics}

Table 1 lists demographic and clinical characteristics of the 105 patients with DM2 and $20 \mathrm{HC}$ subjects included in the study. The median duration of diabetes in patients with DPN-P (10 years) or DPN-NoP (15 years) was longer than in patients w/o DPN (5 years; $P<0.05$ ), although 7 patients w/o DPN were 
diagnosed with DM2 more than 10 years ago. Hemoglobin A1c (HbA1c) values did not significantly differ between patient groups. As would be expected, patients with DPN-P had higher DNS and Toronto CNS symptom scores than patients with DPN-NoP $(P<0.001)$. The majority of patients with DM2 had a family history of diabetes. Half of the patients with DPN-P further reported a family member with neuropathy, a larger proportion than that of patients with DPN-NoP or DM2 w/o DPN $(P<0.001)$.

\subsection{Neuropathic phenotypes}

The outcome of the standardized assessment was in good agreement with the independent neurological triage of patients with and without DPN and patients with neuropathic pain. Relative to age-adjusted reference values [41], the assessment revealed increased pain sensitivity in 68 subjects and a loss of sensory function in 86-81 subjects. Sensory deficits were also found in $3 \mathrm{HC}$ subjects and 3 patients classified as having DM2 w/o DPN (Fig. 1).

Patients with DPN-P described both pain located in the skin and deeper (Fig. 2). Intermittent episodes of spontaneous or stimulus-dependent pain were more common than continuous pain, which was reported by $34 \%$ of the patients. Sharp, stinging or pins and needles were the most frequent pain descriptions. Burning pain, often regarded as a pathognomonic symptom of neuropathic pain, was reported by $64 \%$ of the patients. Paresthesia occurred in virtually all patients with DPN-P and $74 \%$ of patients with DPN-NoP. Tingling was the predominant type of paresthesia. Patients with DPN-P further described paresthesia of other sensory qualities, for example itching or warm, which were rare in DPNNoP $(P \leq 0.015)$ (Fig. 2). The overall pattern of sensory deficits in DPN-P and DPN-NoP was similar, although the responses to some mechanical stimuli were more often decreased in patients with a painful neuropathy. For example, the detection of brush movement was reduced in $39 \%$ of patients with DPN-P versus $5 \%$ of patients with DPN-NoP $(P=0.004)$ (Fig. 3).

Increased excitability of nerve fibers whose terminals are spared from degenerative changes or regenerating can be a critical modulator of neuropathic pain $[17,27,35,36]$. We identified 7 patients with DPN-P who, based on their physical examination, appeared to have intact small-fiber function. They 
accurately responded to pinprick, were able to discriminate cold and hot temperatures, and did not exhibit abnormal sweating $[21,55]$. All of these patients described stinging pain or a sensation of painful needles and pins. Burning (43\%) or cold pain (14\%) was less frequent compared to patients with deficits in small fiber function, whereas temporal summation (43\%), pinprick (57\%) and heat hyperalgesia (71\%) were more common. We also identified 5 patients with DPN-P who did not show clinical signs of large-fiber dysfunction. These patients were able to detect light punctate stimuli $(2 \mathrm{~g})$, brush movement and vibration, and had intact proprioception. Interestingly none of these patients described a continuous presence of pain (Fig. 2). Light punctate or dynamic mechanical stimulation did not evoke a painful response in patients with preserved large-fiber function (Fig. 3).

\subsection{Correlations between symptoms and signs}

Numbness was a common symptom among all patients with DPN. Numbness is typically associated with a deficit in touch sensitivity [32]. Physical tests revealed indeed a decreased detection of mechanical stimuli in the majority of patients reporting numbness. However, some patients experienced numbness in the absence of a clinically manifest touch deficit. Six patients showed only a reduced sense of vibration. Seven patients had decreased responses only in nociceptive tests and in 4 of these patients, only the responses to noxious cold or hot temperature were affected (Fig. 4A). Rather than indicating a particular sensory deficit, numbness may signal more generally a disruption of the normal pattern of afferent input.

We evaluated the relation between symptoms and physical test outcomes further by conducting a PCA. Painful responses to light mechanical stimulation $(2 \mathrm{~g})$ showed good correlation with a history of mechanical allodynia $(R=0.44)$. Rather unexpected was the weak correlation of painful responses to brush movement $(R=0.24)$. Patients reporting increased sensitivity to pressure-evoked pain, for example, from a tight shoe, also responded with pain to the application of calibrated pressure in the physical examination $(R=0.40)$, whereas mechanical stimulation at lighter force was painless (Fig. 4B). Patient reports of thermal allodynia equally correlated with painful responses to cold or warm stimuli in the physical tests (Fig. 4C). In addition, heightened temperature sensitivity appeared to influence the 
sensory character of pain and paresthesia. Patients with cold-evoked pain described pain $(R=0.44)$ or paresthesia $(R=0.38)$ of a cold quality more often than burning pain $(R=0.09)$ or warm paresthesia $(R$ $=0.20)($ Fig. 4C) .

Deficits in sensory function correlated across mechanical test modalities, whereas stimulusdependent pain appeared to be more specific. For example, allodynia in response to light punctate stimulation was strongly associated with pain evoked by brush movement $(R=0.59)$ but distinct from pinprick hyperalgesia ( $R=0.11$ and -0.02 , respectively) (Fig. 5A). Painful responses to cold or warm stimuli also showed greater temperature specificity than deficits in temperature discrimination (Fig. 5B).

\subsection{Principal components of the phenotypic variance}

$\underline{\text { PCA reduces the complexity of multivariate outcomes to principal components and calculates the loading }}$ (weight) of each variable on these components. Loss of sensory function and pain hypersensitivity determined the first two principal components of phenotypic variance in the physical tests (Fig. 6A). Thermal test responses scored higher on the first component, whereas responses to punctate mechanical stimulation or pinprick dominated in the second component (Fig. 6B, C). These two components accounted for $16 \%$ and $12 \%$, respectively, of the variance. A third component $(7 \%)$ was defined by differences in the temporal summation of repeated mechanical stimulation and autonomic signs (data not shown). Across 11 components, which combined accounted for $70 \%$ of the variance between physical signs, we identified a decrease in the detection of warm temperature $\left(40^{\circ} \mathrm{C}\right)$, a reduced response to pinprick, loss of proprioception, abnormal sweating and trophic changes of the skin as indicators of DPN with consistently high weights. Heat hyperalgesia $\left(45^{\circ} \mathrm{C}\right)$ and temporal summation of mechanical stimuli were signs of increased pain sensitivity with high loadings on the components. We also identified tests that contributed little to the phenotypic variance. For example, a painful response to stimulation with the von Frey filament of $2-g$ strength was strongly correlated to pain evoked by the filament of 26-g strength (Fig. 6). Hence we considered the test redundant.

Differences in the experience of pain caused by a certain body position or physical activity such 
as walking, and the sensitivity to cold or warm temperatures marked the first principal component of neuropathic symptoms (Fig. 7). Patient reports of pain evoked by these external factors were responsible for $39 \%$ of the variance. The sensory quality of pain or paresthesia was a prominent factor on the second principal component, which accounted for $6 \%$ of the phenotypic variance (data not shown). Across 9 symptom components, which combined accounted for $70 \%$ of the phenotypic variance, we found high weights for the presence of continuous versus episodic pain, the experience of cold-evoked pain, a burning quality of pain, and paresthesia characterized by a cold or pulling sensation. On the other hand, symptoms such as throbbing pain or pain that felt like an electric shock did not add substantially to the differentiation of phenotypes.

\subsection{Standardized Evaluation of Pain and Somatosensory Function (StEPS)}

We combined items with high weights on the principal components and items such as tingling paresthesia or numbness, which were common symptoms across all phenotypes of DPN, in an abridged assessment tool that we named Standardized Evaluation of Pain and Somatosensory Function (StEPS). StEPS includes 20 interview questions (plus a global intensity rating of current pain) and 20 physical tests (see Supplemental Digital Content 1). We combined items with high loadings on the principal components in a reduced set of interview questions and physical tests and named this abridged assessment tool Standardized Evaluation of Pain and Somatosensory Function (StEPS) (see Supplemental Digital Content 1). StEPS generates a brief but informative phenotypic profile of sensory neuropathy that can be completed within 20 minutes. The intensity of spontaneous pain and painful responses to external stimuli are rated independently to document their specific contribution to the patient's experience of pain. To compare the diagnostic performance of StEPS with that of the original 47-item interview and the 39item physical examination, we conducted a receiver operating characteristic (ROC) analysis. StEPS clearly distinguished DPN-P (area under the ROC curve 1.00; Cl 0.99, 1.00) and DPN-NoP $(0.89 ; \mathrm{Cl}$ $0.79,0.99)$ from DM2 w/o DPN_(Fig. 8). StEPS also differentiated DPN-P from DPN-NoP with high sensitivity and specificity $(0.98 ; \mathrm{Cl} 0.96,1.00)$. Numbness, tingling paresthesia, a decreased response to 
pinprick and a reduced sense of vibration were among the most sensitive and specific symptoms or signs, respectively, of neuropathy. Deep or superficial pain, a stinging or burning pain quality, episodic pain and painful aftersensations were among the strongest indicators of DPN-P (see Table S1, Supplemental Digital Content 2). The diagnostic accuracy of StEPS was equivalent to that of the full assessment protocol (Fig. 8). Sensitivity and specificity of the pain diagnosis matched or exceeded those of the DN4 questionnaire (see Table $\underline{\text { S2}}$, Supplemental Digital Content $2 \underline{3}$ ).

\section{Discussion}

We developed a novel phenotyping tool for painful peripheral neuropathy based on an analysis of interindividual-differences in the clinical manifestation of DPN. StEPS utilizes a standardized bedside assessment of symptoms and signs to reveal deficits in sensory functions and determine the essential characteristics of neuropathic pain. Instead of measuring a global average pain score, StEPS obtains records separate pain ratings to evaluate, for example, the specific intensity of dynamic mechanical or cold allodynia. Thus treatment response can be evaluated with greater precision and examined for potential associations with the clinical phenotype. StEPS generates more detailed information than screening tools and questionnaires that are primarily designed to distinguish neuropathic from nociceptive pain [8]. Physical tests included in the phenotyping protocol examine concrete peripheral nerve functions as opposed to assessing the severity of neuropathy by measuring the impairment of daily activities. Designed for a bedside evaluation of patients, StEPS offers a pragmatic and affordable alternative to QST that will be easier to implement in clinical trials with large numbers of patients or multiple participating centers.

Our phenotyping strategy included-comprised two complementary components, a structured patient interview and a physical examination involving standardized test stimuli. Patient descriptions of the sensory quality of pain or paresthesia provided clues as to which subtypes of neurons were affected by DPN-P. Consistent with previous findings [6], stinging or pain and painful-pins and needles were the most common characterizations of pain, an observation that corresponds to previous findings $\{$ Baron, 
$2009,19592166\}$. Psychophysiological studies have shown that pain of this quality originates in Aס fibers, which are typically activated by punctate stimuli such as pinprick $[40,65]$. Burning pain, which is elicited by the activation of heat-sensitive $\mathrm{C}$ fibers $[44,49]$, was present in approximately two thirds of the patients with DPN-P but less common in a subgroup of patients with clinically intact small-fiber function.

Several sensory qualities of paresthesia were almost exclusively reported by patients with DPN$\underline{P}$. These included painless warm or cold sensations, which may indicate an increased activity of

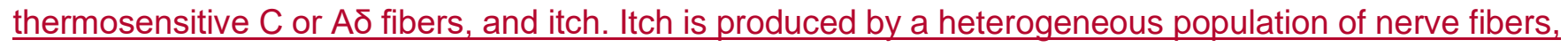
some of which may also be nociceptive. The association with DPN-P may therefore reflect a heightened excitability of polymodal afferents that signal both itch and pain [7]. However, although the sensory characteristics of pain and paresthesia appeared to indicate the differentialinvolvement of different nerve fiber populations, theirthe overall contribution of these symptoms to the phenotypic variance in DPN-was small (6\%). Patients typically used multiple attributes to describe their pain and paresthesia, which reduced the selectivity of these characteristics.-compared to differences in the sensitivity to painprovoking stimuli (39\%).

Numbness was the most frequent painless symptom. Patients in this study reported numbness more often than participants in previous surveys \{Baron, 2009, 19592166;0tto, 2003, 12507713\}. Unlike positive sensations such as tingling paresthesia, numbness is usually associated with a loss of touch detection. Mechanosensitivity was reduced in the majority of patients who described numbness of their feet. However, in some patients only the sense of vibration or the response to pinprick was decreased, whereas the detection of light mechanical stimuli was intact. Eight patients did not show a mechanosensory deficit in the physical tests. We did not measure exact thresholds of mechanical stimulus detection. Consequently, we can only draw conclusions on the relation between numbness and elinical deficits in mechanosensitivity that would have been recognizable in the bedside tests we performed. On the other hand, QST studies confirm that some patients with polyneuropathy experience numbness or "discomfort" in their hands and feet in the absence of touch deficits fHershman, 2011, 21128110\}. Numbness even occurs in conditions of chronic non-neuropathic pain, which has led to the 
hypothesis that plasticity in central sensory pathways contributes to its development fGeber, 2008, 18423989\}. More research is needed to resolve the relation between the perception of numbness and disturbances in the normal pattern of afferent input.

Experience of evoked pain in response to mechanical or thermal stimuli and painful aftersensations defined the largest proportion of phenotypic differences in the interview. The analysis of physical test outcomes confirmed that stimulus-dependent pain distinguished better between patient phenotypes than sensory loss. However, the majority of patients with DPN-P exhibited sensory deficits including those indicative of small-fiber degeneration, Cheng, 2013, 23685187; Devigili, 2008, 18524793\}. Sensory loss was actually more frequent in DPN-P than DPN-NoP. Mechanosensory Sensory deficits correlated across different test modalities, whereas painful responses, for example, to the-punctate stimulation with von Frey filaments, brush movement or pinprick hyperalgesia-showed greater specificity. We found a similar pattern of correlated sensory deficits but distinct painful reactions when we tested the response to thermal stimuli.This pattern matches the findings of a recent meta-analysis by Freeman and colleagues, who revisited QST results from four randomized clinical trials involving patients with painful DPN, polyneuropathy associated with human immunodeficiency virus (HIV) infection, nerve trauma or central poststroke pain. In this study, Increased discriminatoryincreases in the detection thresholds for mechanical or thermal stimuli were common and highly correlated, whereas allodynia and hyperalgesia were more discriminating differed between stimulus modalities-[29]. Studies-Previous investigations that combined QST, skin biopsies and nerve conduction tests provide further evidence that degenerative changes in DPN usually affect several types of nerve fibers, leading to a explaining the-broad spectrum of deficits with little variation between patients [51]. Evoked pain distinguishes between patient phenotypes better than sensory loss.

Low specificity of sensory deficits was one factor contributing to the relatively weak separation between DPN-P and DPN-NoP when the outcome of the physical tests alone was considered. Painful $\underline{\text { responses to light touch, brush movement, moderately cold or warm temperature were found exclusively }}$ in patients with DPN-P and provided for a better distinction. However, fewer than half of the patients with 
DPN-P in our study exhibited mechanical or thermal allodynia. For example, punctate mechanical allodynia was present in $7 \%$ of patients with DPN-P. This proportion was smaller than that observed in a previous examination of 35 patients with DPN [45], but comparable to the percentage of patients who described moderate or severe pain in response to the stimulation with von Frey filaments in four larger QST investigations [29]. The frequency of hyperalgesia evoked by pinprick or cold was also similar to that in previous QST studies of DPN \{Freeman, 2014, 24472518\}. In order to achieve high sensitivity and specificity for the identification of neuropathic pain phenotypes, physical tests need to be combined with an interview. Otherwise, critical aspects of the phenotype will be missed: spontaneous pain, the sensory quality of pain or paresthesia, and the patient's experience of evoked pain during daily activities, which standard stimuli in a test setting can only incompletely reproduce.

A phenotype marked by the combination of continuous pain, a stinging pain quality, hyperalgesia in response to pinprick or heat, increased pain sensitivity to pinprick, pressure and heat, and temporal summation suggested heightened excitability of nociceptive nerve fibers in a patient subgroup with clinically intact small-fiber function. Similar associations of ongoing pain and mechanical or thermal hyperalgesia have been reported in patients with postherpetic neuralgia or chronic pain after nerve trauma $[33,47]$. Preserved skin innervation and thermal thresholds support the concept that "irritable" nociceptive afferents are causing pain in patients with these clinical and histopathological characteristics [48]. DPN certainly involves additional mechanisms of spontaneous and evoked pain, because similar $\underline{\text { symptoms and signs can be observed in patients with impaired small-fiber functions. }}$

Based onWe used a PCA to determine correlation patterns of symptoms and signs and identify the principal components of phenotypic diversity in DPN., we identified those items of the structured interview and the physical examination that best captured the phenotypic diversity of DPN. We combined these items in StEPS to create We combined interview items and physical tests with high weight on these components to create StEPS. StEPS is a bedside tool for the assessment of painful neuropathy whose application does not require major technical equipment or advanced expertise. Separate intensity ratings for painful symptoms and signs allow measuring changes in the pain phenotype over the course of the 
disease and evaluating the response to treatment in greater detail than global pain scores. Some of the items in StEPS are also contained in screening tools for neuropathic pain such as DN4 or painDETECT [10,31]. These items reflect core features of neuropathic pain that contribute to thealso apply to phenotypes of DPN, for example, a burning character quality of pain or pain evoked by brush movement [8]. StEPS is more comprehensive than the Neuropathic Pain Symptom Inventory and other questionnaires designed for self-administration that have previously been used to cluster patients with neuropathic pain based on combination patterns of sensory abnormalities $[6,11,29]$. The inclusion of physical tests in StEPS increases the depth and specificity-of the phenotype characterization and enables the validation of patient-reported painful stimuli [61]. Standardization of the assessment procedure is essential. Dyck and colleagues have demonstrated the limits of evaluating DPN without a defined protocol, even when patient interview and examination are performed by an experienced physician [25]. StEPS does not yield quantitative information about thresholds of sensory discrimination or pain. This is the domain of QST [4,52]. However, QST relies entirely on the recording of stimulus-evoked responses. The technique does not assess spontaneous pain or paresthesia, which constitute relevant components of DPN phenotypes. As a consequence, QST alone is not always sufficient to distinguish painful from painless neuropathies [21,34,54].

The phenotypic diversity of DPN reflects differences in the vulnerability of distinct nerve fiber populations to hyperglycemia-induced damage and other pathogenetic factors of neuropathy in DM2. It furthermore suggests differences between patients in the pathophysiological mechanisms responsible for neuropathic pain in the mechanisms responsible for the development of pain, even among patients who, based on the extent of sensory deficits, exhibit similar levels of neuropathy $[18,62]$. Identifying pain mechanisms in patients is challenging, but clinical symptoms and signs can provide surrogate markers of the underlying pathophysiological processes to that can guide treatment decisions. Correlations between sensory abnormalities and analgesic treatment response have, for example, been reported for oxcarbazepine, topical clonidine and botulinum toxin $A[16,20,46]$. StEPS facilitates the collection of structured phenotypic information. Compared to QST, a bedside tool like StEPS is less expensive and 
easier to administer in large treatment trials or genetic studies with multiple participating centers. Phenotypic characteristics of patients can be established in clinical practice without the need to invest in extensive training or major equipment. The sSensitivity of StEPS to treatment response and its utility to phenotype patients with neuropathies other than DPN will be tested in future studies. We hope that this pragmatic phenotyping strategy is going to advance the development of tailored pharmacotherapeutic approaches to neuroprotection and pain treatment.

\section{Acknowledgments}

This work was supported by an unrestricted research grant from GlaxoSmithKline.

\section{Conflict of interest statement}

J. Scholz has received consultation fees from GlaxoSmithKline. J. B. Davis and S. N. Tate are former employees and shareholders of GlaxoSmithKline.

\section{List of Supplemental Digital Content}

Supplemental Digital Content 1. Standardized Evaluation of Pain and Somatosensory Function (StEPS). pdf

Supplemental Digital Content 2. Table listing the area under the ROC curves for individual StEPS items in the distinction between patients with diabetes who did not develop a neuropathy (DM2 w/o DPN) and patients with painful $(P)$ or painless (NoP) variants of DPN. docx

Supplemental Digital Content $\underline{2} \underline{3}$. Table comparing the sensitivity and specificity to identify DPN-P of the full version of the assessment protocol, StEPS and DN4. docx

\section{References}


[1] Abbott CA, Malik RA, van Ross ER, Kulkarni J, Boulton AJ. Prevalence and characteristics of painful diabetic neuropathy in a large community-based diabetic population in the U.K. Diabetes Care 2011;34(10):2220-2224.

[2] American Diabetes Association. Diagnosis and classification of diabetes mellitus. Diabetes Care 2009;32 Suppl 1:S62-67.

[3] Andreassen CS, Jakobsen J, Andersen H. Muscle weakness: a progressive late complication in diabetic distal symmetric polyneuropathy. Diabetes 2006;55(3):806-812.

[4] Backonja MM, Attal N, Baron R, Bouhassira D, Drangholt M, Dyck PJ, Edwards RR, Freeman R, Gracely R, Haanpaa MH, Hansson P, Hatem SM, Krumova EK, Jensen TS, Maier C, Mick G, Rice AS, Rolke R, Treede RD, Serra J, Toelle T, Tugnoli V, Walk D, Walalce MS, Ware M, Yarnitsky D, Ziegler D. Value of quantitative sensory testing in neurological and pain disorders: NeuPSIG consensus. Pain 2013;154(9):1807-1819.

[5] Baron R, Forster M, Binder A. Subgrouping of patients with neuropathic pain according to painrelated sensory abnormalities: a first step to a stratified treatment approach. The Lancet Neurology 2012;11(11):999-1005.

[6] Baron R, Tolle TR, Gockel U, Brosz M, Freynhagen R. A cross-sectional cohort survey in 2100 patients with painful diabetic neuropathy and postherpetic neuralgia: Differences in demographic data and sensory symptoms. Pain 2009.

[7] Bautista DM, Wilson SR, Hoon MA. Why we scratch an itch: the molecules, cells and circuits of itch. Nature neuroscience 2014;17(2):175-182.

[8] Bennett MI, Attal N, Backonja MM, Baron R, Bouhassira D, Freynhagen R, Scholz J, Tolle TR, Wittchen HU, Jensen TS. Using screening tools to identify neuropathic pain. Pain 2007;127(3):199-203.

[9] Bierhaus A, Fleming T, Stoyanov S, Leffler A, Babes A, Neacsu C, Sauer SK, Eberhardt M, Schnolzer M, Lasitschka F, Neuhuber WL, Kichko TI, Konrade I, Elvert R, Mier W, Pirags V, Lukic IK, Morcos M, Dehmer T, Rabbani N, Thornalley PJ, Edelstein D, Nau C, Forbes J, Humpert PM, 
Schwaninger M, Ziegler D, Stern DM, Cooper ME, Haberkorn U, Brownlee M, Reeh PW, Nawroth PP. Methylglyoxal modification of Nav1.8 facilitates nociceptive neuron firing and causes hyperalgesia in diabetic neuropathy. Nat Med 2012;18(6):926-933.

[10] Bouhassira D, Attal N, Alchaar H, Boureau F, Brochet B, Bruxelle J, Cunin G, Fermanian J, Ginies P, Grun-Overdyking A, Jafari-Schluep H, Lanteri-Minet M, Laurent B, Mick G, Serrie A, Valade D, Vicaut E. Comparison of pain syndromes associated with nervous or somatic lesions and development of a new neuropathic pain diagnostic questionnaire (DN4). Pain 2005;114(1-2):2936.

[11] Bouhassira D, Attal N, Fermanian J, Alchaar H, Gautron M, Masquelier E, Rostaing S, LanteriMinet M, Collin E, Grisart J, Boureau F. Development and validation of the Neuropathic Pain Symptom Inventory. Pain 2004;108(3):248-257.

[12] Bouhassira D, Wilhelm S, Schacht A, Perrot S, Kosek E, Cruccu G, Freynhagen R, Tesfaye S, Lledo A, Choy E, Marchettini P, Mico JA, Spaeth M, Skljarevski V, Tolle T. Neuropathic pain phenotyping as a predictor of treatment response in painful diabetic neuropathy: Data from the randomized, double-blind, COMBO-DN study. Pain 2014.

[13] Bril V, Perkins BA. Validation of the Toronto Clinical Scoring System for diabetic polyneuropathy. Diabetes Care 2002;25(11):2048-2052.

[14] Bril V, Tomioka S, Buchanan RA, Perkins BA. Reliability and validity of the modified Toronto Clinical Neuropathy Score in diabetic sensorimotor polyneuropathy. Diabet Med 2009;26(3):240246.

[15] Callaghan BC, Cheng HT, Stables CL, Smith AL, Feldman EL. Diabetic neuropathy: clinical manifestations and current treatments. Lancet Neurol 2012;11(6):521-534.

[16] Campbell CM, Kipnes MS, Stouch BC, Brady KL, Kelly M, Schmidt WK, Petersen KL, Rowbotham MC, Campbell JN. Randomized control trial of topical clonidine for treatment of painful diabetic neuropathy. Pain 2012. 
[17] Cheng HT, Dauch JR, Porzio MT, Yanik BM, Hsieh W, Smith AG, Singleton JR, Feldman EL. Increased axonal regeneration and swellings in intraepidermal nerve fibers characterize painful phenotypes of diabetic neuropathy. The Journal of Pain 2013;14(9):941-947.

[18] Costigan M, Scholz J, Woolf CJ. Neuropathic pain: a maladaptive response of the nervous system to damage. Annu Rev Neurosci 2009;32:1-32.

[19] Daousi C, MacFarlane IA, Woodward A, Nurmikko TJ, Bundred PE, Benbow SJ. Chronic painful peripheral neuropathy in an urban community: a controlled comparison of people with and without diabetes. Diabet Med 2004;21(9):976-982.

[20] Demant DT, Lund K, Vollert J, Maier C, Segerdahl M, Finnerup NB, Jensen TS, Sindrup SH. The effect of oxcarbazepine in peripheral neuropathic pain depends on pain phenotype: a randomised, double-blind, placebo-controlled phenotype-stratified study. Pain 2014;155(11):2263-2273.

[21] Devigili G, Tugnoli V, Penza P, Camozzi F, Lombardi R, Melli G, Broglio L, Granieri E, Lauria G. The diagnostic criteria for small fibre neuropathy: from symptoms to neuropathology. Brain 2008;131(Pt 7):1912-1925.

[22] Dworkin RH, Turk DC, Peirce-Sandner S, He H, McDermott MP, Farrar JT, Katz NP, Lin AH, Rappaport BA, Rowbotham MC. Assay sensitivity and study features in neuropathic pain trials: an ACTTION meta-analysis. Neurology 2013;81(1):67-75.

[23] Dyck PJ, Davies JL, Litchy WJ, O'Brien PC. Longitudinal assessment of diabetic polyneuropathy using a composite score in the Rochester Diabetic Neuropathy Study cohort. Neurology 1997;49(1):229-239.

[24] Dyck PJ, Kratz KM, Karnes JL, Litchy WJ, Klein R, Pach JM, Wilson DM, O'Brien PC, Melton LJ, 3rd, Service FJ. The prevalence by staged severity of various types of diabetic neuropathy, retinopathy, and nephropathy in a population-based cohort: the Rochester Diabetic Neuropathy Study. Neurology 1993;43(4):817-824.

[25] Dyck PJ, Overland CJ, Low PA, Litchy WJ, Davies JL, O'Brien PC, Albers JW, Andersen H, Bolton CF, England JD, Klein CJ, Llewelyn JG, Mauermann ML, Russell JW, Singer W, Smith AG, 
Tesfaye S, Vella A. Signs and symptoms versus nerve conduction studies to diagnose diabetic sensorimotor polyneuropathy: Cl vs. NPhys trial. Muscle Nerve 2010;42(2):157-164.

[26] Feldman EL, Stevens MJ, Thomas PK, Brown MB, Canal N, Greene DA. A practical two-step quantitative clinical and electrophysiological assessment for the diagnosis and staging of diabetic neuropathy. Diabetes Care 1994;17(11):1281-1289.

[27] Fields HL, Rowbotham M, Baron R. Postherpetic neuralgia: irritable nociceptors and deafferentation. Neurobiol Dis 1998;5(4):209-227.

[28] Finnerup NB, Attal N, Haroutounian S, McNicol E, Baron R, Dworkin RH, Gilron I, Haanpaa M, Hansson P, Jensen TS, Kamerman PR, Lund K, Moore A, Raja SN, Rice AS, Rowbotham M, Sena E, Siddall P, Smith BH, Wallace M. Pharmacotherapy for neuropathic pain in adults: a systematic review and meta-analysis. The Lancet Neurology 2015;14(2):162-173.

[29] Freeman R, Baron R, Bouhassira D, Cabrera J, Emir B. Sensory profiles of patients with neuropathic pain based on the neuropathic pain symptoms and signs. Pain 2014;155(2):367-376.

[30] Freeman R, Chapleau MW. Testing the autonomic nervous system. Handb Clin Neurol 2013;115:115-136.

[31] Freynhagen R, Baron R, Gockel U, Tolle TR. painDETECT: a new screening questionnaire to identify neuropathic components in patients with back pain. Curr Med Res Opin 2006;22(10):1911-1920.

[32] Geber C, Magerl W, Fondel R, Fechir M, Rolke R, Vogt T, Treede RD, Birklein F. Numbness in clinical and experimental pain--a cross-sectional study exploring the mechanisms of reduced tactile function. Pain 2008;139(1):73-81.

[33] Gottrup H, Nielsen J, Arendt-Nielsen L, Jensen TS. The relationship between sensory thresholds and mechanical hyperalgesia in nerve injury. Pain 1998;75(2-3):321-329.

[34] Hansson P, Backonja M, Bouhassira D. Usefulness and limitations of quantitative sensory testing: clinical and research application in neuropathic pain states. Pain 2007;129(3):256-259. 
[35] Haroutounian S, Nikolajsen L, Bendtsen TF, Finnerup NB, Kristensen AD, Hasselstrom JB, Jensen TS. Primary afferent input critical for maintaining spontaneous pain in peripheral neuropathy. Pain 2014;155(7):1272-1279.

[36] Kleggetveit IP, Namer B, Schmidt R, Helas T, Ruckel M, Orstavik K, Schmelz M, Jorum E. High spontaneous activity of C-nociceptors in painful polyneuropathy. Pain 2012;153(10):2040-2047.

[37] Lefaucheur JP, Creange A. Neurophysiological testing correlates with clinical examination according to fibre type involvement and severity in sensory neuropathy. $\mathrm{J}$ Neurol Neurosurg Psychiatry 2004;75(3):417-422.

[38] Loken LS, Wessberg J, Morrison I, McGlone F, Olausson H. Coding of pleasant touch by unmyelinated afferents in humans. Nature neuroscience 2009;12(5):547-548.

[39] Low PA, Benrud-Larson LM, Sletten DM, Opfer-Gehrking TL, Weigand SD, O'Brien PC, Suarez GA, Dyck PJ. Autonomic symptoms and diabetic neuropathy: a population-based study. Diabetes Care 2004;27(12):2942-2947.

[40] Mackenzie RA, Burke D, Skuse NF, Lethlean AK. Fibre function and perception during cutaneous nerve block. Journal of neurology, neurosurgery, and psychiatry 1975;38(9):865-873.

[41] Magerl W, Krumova EK, Baron R, Tolle T, Treede RD, Maier C. Reference data for quantitative sensory testing (QST): refined stratification for age and a novel method for statistical comparison of group data. Pain 2010;151(3):598-605.

[42] Maier C, Baron R, Tolle TR, Binder A, Birbaumer N, Birklein F, Gierthmuhlen J, Flor H, Geber C, Huge V, Krumova EK, Landwehrmeyer GB, Magerl W, Maihofner C, Richter H, Rolke R, Scherens A, Schwarz A, Sommer C, Tronnier V, Uceyler N, Valet M, Wasner G, Treede RD. Quantitative sensory testing in the German Research Network on Neuropathic Pain (DFNS): somatosensory abnormalities in 1236 patients with different neuropathic pain syndromes. Pain 2010;150(3):439450. 
[43] Meijer JW, Smit AJ, Sonderen EV, Groothoff JW, Eisma WH, Links TP. Symptom scoring systems to diagnose distal polyneuropathy in diabetes: the Diabetic Neuropathy Symptom score. Diabet Med 2002;19(11):962-965.

[44] Ochoa J, Torebjork E. Sensations evoked by intraneural microstimulation of $C$ nociceptor fibres in human skin nerves. The Journal of physiology 1989;415:583-599.

[45] Otto M, Bak S, Bach FW, Jensen TS, Sindrup SH. Pain phenomena and possible mechanisms in patients with painful polyneuropathy. Pain 2003;101(1-2):187-192.

[46] Ranoux D, Attal N, Morain F, Bouhassira D. Botulinum toxin type A induces direct analgesic effects in chronic neuropathic pain. Annals of neurology 2008;64(3):274-283.

[47] Rowbotham MC, Fields HL. The relationship of pain, allodynia and thermal sensation in postherpetic neuralgia. Brain 1996;119 ( Pt 2):347-354.

[48] Rowbotham MC, Yosipovitch G, Connolly MK, Finlay D, Forde G, Fields HL. Cutaneous innervation density in the allodynic form of postherpetic neuralgia. Neurobiol Dis 1996;3(3):205214.

[49] Schmelz M, Schmid R, Handwerker HO, Torebjork HE. Encoding of burning pain from capsaicintreated human skin in two categories of unmyelinated nerve fibres. Brain : a journal of neurology 2000;123 Pt 3:560-571.

[50] Scholz J, Mannion RJ, Hord DE, Griffin RS, Rawal B, Zheng H, Scoffings D, Phillips A, Guo J, Laing RJ, Abdi S, Decosterd I, Woolf CJ. A novel tool for the assessment of pain: validation in low back pain. PLoS Med 2009;6(4):e1000047.

[51] Shun CT, Chang YC, Wu HP, Hsieh SC, Lin WM, Lin YH, Tai TY, Hsieh ST. Skin denervation in type 2 diabetes: correlations with diabetic duration and functional impairments. Brain 2004;127(Pt 7):1593-1605.

[52] Shy ME, Frohman EM, So YT, Arezzo JC, Cornblath DR, Giuliani MJ, Kincaid JC, Ochoa JL, Parry GJ, Weimer LH. Quantitative sensory testing: report of the Therapeutics and Technology 
Assessment Subcommittee of the American Academy of Neurology. Neurology 2003;60(6):898904.

[53] Singleton JR, Bixby B, Russell JW, Feldman EL, Peltier A, Goldstein J, Howard J, Smith AG. The Utah Early Neuropathy Scale: a sensitive clinical scale for early sensory predominant neuropathy. Journal of the peripheral nervous system : JPNS 2008;13(3):218-227.

[54] Sorensen L, Molyneaux L, Yue DK. The level of small nerve fiber dysfunction does not predict pain in diabetic Neuropathy: a study using quantitative sensory testing. Clin J Pain $2006 ; 22(3): 261-265$.

[55] Tesfaye S, Boulton AJ, Dyck PJ, Freeman R, Horowitz M, Kempler P, Lauria G, Malik RA, Spallone V, Vinik A, Bernardi L, Valensi P. Diabetic neuropathies: update on definitions, diagnostic criteria, estimation of severity, and treatments. Diabetes Care 2010;33(10):2285-2293.

[56] Tesfaye S, Chaturvedi N, Eaton SE, Ward JD, Manes C, lonescu-Tirgoviste C, Witte DR, Fuller JH. Vascular risk factors and diabetic neuropathy. The New England journal of medicine $2005 ; 352(4): 341-350$.

[57] Treede RD, Jensen TS, Campbell JN, Cruccu G, Dostrovsky JO, Griffin JW, Hansson P, Hughes R, Nurmikko T, Serra J. Neuropathic pain: redefinition and a grading system for clinical and research purposes. Neurology 2008;70(18):1630-1635.

[58] Treede RD, Rief W, Barke A, Aziz Q, Bennett MI, Benoliel R, Cohen M, Evers S, Finnerup NB, First MB, Giamberardino MA, Kaasa S, Kosek E, Lavand'homme P, Nicholas M, Perrot S, Scholz J, Schug S, Smith BH, Svensson P, Vlaeyen JW, Wang SJ. A classification of chronic pain for ICD-11. Pain 2015;156(6):1003-1007.

[59] Truini A, Biasiotta A, Di Stefano G, Leone C, La Cesa S, Galosi E, Piroso S, Pepe A, Giordano C, Cruccu G. Does the epidermal nerve fibre density measured by skin biopsy in patients with peripheral neuropathies correlate with neuropathic pain? Pain 2014;155(4):828-832.

[60] Truini A, Garcia-Larrea L, Cruccu G. Reappraising neuropathic pain in humans--how symptoms help disclose mechanisms. Nature reviews Neurology 2013;9(10):572-582. 
[61] van Hecke O, Kamerman PR, Attal N, Baron R, Bjornsdottir G, Bennett DL, Bennett MI, Bouhassira D, Diatchenko L, Freeman R, Freynhagen R, Haanpaa M, Jensen TS, Raja SN, Rice AS, Seltzer Z, Thorgeirsson TE, Yarnitsky D, Smith BH. Neuropathic pain phenotyping by international consensus (NeuroPPIC) for genetic studies: a NeuPSIG systematic review, Delphi survey, and expert panel recommendations. Pain 2015;156(11):2337-2353.

[62] von Hehn CA, Baron R, Woolf CJ. Deconstructing the neuropathic pain phenotype to reveal neural mechanisms. Neuron 2012;73(4):638-652.

[63] Young MJ, Boulton AJ, MacLeod AF, Williams DR, Sonksen PH. A multicentre study of the prevalence of diabetic peripheral neuropathy in the United Kingdom hospital clinic population. Diabetologia 1993;36(2):150-154.

[64] Ziegler D, Gries FA, Spuler M, Lessmann F. The epidemiology of diabetic neuropathy. Diabetic Cardiovascular Autonomic Neuropathy Multicenter Study Group. J Diabetes Complications $1992 ; 6(1): 49-57$

[65] Ziegler EA, Magerl W, Meyer RA, Treede RD. Secondary hyperalgesia to punctate mechanical stimuli. Central sensitization to A-fibre nociceptor input. Brain : a journal of neurology 1999;122 ( Pt 12):2245-2257. 


\section{Figure legends}

Figure 1. Standards for the Reporting of Diagnostic Accuracy (STARD) flowchart. Numbers marked with an asterisk $\left(^{*}\right)$ include 60 subjects who exhibited both increased pain sensitivity and sensory deficits.

Figure 2. Frequency distribution for selected neuropathic symptoms. We used Fisher's exact test to calculate the statistical significance of differences between diabetic polyneuropathy (DPN) with (P) and without pain (NoP). $P$ values of significant differences are shown only when symptoms were present in either patient group. Separate frequency charts show the symptoms of 7 patients with DPN-P who, based on their physical examination, had intact small-fiber functions (response to pinprick, temperature discrimination, sweating) and 5 patients with DPN-NoP who had clinically intact large-fiber functions (detection of light punctate stimuli, brush movement or vibration, senses of position and passive movement; see also Figure 3).

Figure 3. Selected outcomes of the standardized physical tests. $P$ values from Fisher's exact test are shown for significant differences between diabetic polyneuropathy (DPN) with (P) and without pain (NoP) when clinical signs were present in either patient group. Seven patients with DPN-P had intact small-fiber functions (response to pinprick, temperature discrimination, sweating). We also identified 5 patients with DPN-P who had intact large-fiber functions (detection of light punctate stimuli, brush movement and vibration, senses of position and passive movement). Temporal summation (1) denotes an initially painless response that turned painful during repeated stimulation. Temporal summation (2) indicates that the first stimulus was painful, but pain intensity increased during repeated stimulation.

Figure 4. Correlations of symptoms and signs. (A) Venn diagrams show the association of numbness with deficits in the detection of innocuous mechanical stimulation (light blue), proprioceptive deficits (purple) or decreased responses to noxious stimulation (orange). Diagram sections contain the numbers 
of patients with the respective combination of numbness and signs. $(\mathrm{B}, \mathrm{C})$ The correlation of symptoms and signs was examined further in a principal component analysis (PCA). Circles are scaled to reflect the proportion of patients exhibiting the respective combination of symptoms or signs. Pearson correlation coefficients are indicated based on the color key. (B) Correlation between the outcomes of selected bedside tests and patient reports of pain evoked by light touch or pressure. For example, $21(84 \%)$ of 25 patients reporting allodynia and $27(79 \%)$ of 34 patients with increased pressure sensitivity experienced painful aftersensations, $R=0.52$ and 0.57 , respectively. (C) Correlation between the sensory character of pain or paresthesia, responses to thermal stimuli and patient reports of cold or warmth-evoked pain. Eleven (65\%) of 17 patients reporting cold-evoked pain described a painful cold sensation $(R=0.44)$, compared to 8 patients $(47 \%)$ describing pain of a burning quality $(R=0.09)$. Twelve patients $(71 \%)$ also described cold paresthesia $(R=0.38)$, whereas none of the patients reported the experience of warm paresthesia.

Figure 5. Correlation matrix of sensory deficits and pain provoked by $(A)$ mechanical or $(B)$ thermal test stimuli. Circle size reflects the proportion of patients exhibiting the respective combination of test outcomes. Pearson correlation coefficients are represented by magenta (negative) and green (positive) colors.

Figure 6. Principal components contributing to the variance of clinical signs among patients. A principal component analysis (PCA) revealed the major dimensions of phenotypic differences among patients with DM2. Each dot on the scatter plots in this figure represents one of the 39 test items in the physical examination. Scales indicate the loading (weight) of items on the respective component. (A) Sensory deficits (white dots), painful responses (dark grey dots) and autonomic signs (light gray dots) are projected onto the first two principal components. Sensory deficits (white) and painful responses (dark grey) determined the first two dimensions of phenotypic differences, with painful responses having greater weight on Component 1, and sensory deficits bearing greater absolute weight on Component 2. 
(B) Responses to thermal stimuli produced the greatest differences on the first componentComponent 1 , which accounted for $16 \%$ of the total variance in physical test outcomes. Pink dots identify responses to cold, red dots responses to warm or hot stimuli. (C) Responses to punctate mechanical stimuli dominated on the second principal component, which contributed $12 \%$ to the overall variance in clinical signs. Test outcomes after stimulation with von Frey filaments are shown in light orange, responses to pinprick in dark orange.

Figure 7. Principal component analysis (PCA) of neuropathic symptoms. Each dot represents one of the 47 items in the interview. The first-leading symptom component was defined by differences in the experience of evoked pain. Light blue dots mark painful reactions to different physical stimuli. Dark blue dots indicate the reported duration of pain relative to the stimulus exposure. This component alone accounted for $39 \%$ of the total variance of symptoms.

Figure 8. Diagnostic performance of the Standardized Evaluation of Pain and Somatosensory Function (StEPS). Receiver operating characteristic (ROC) curves indicate the sensitivity and specificity of StEPS to identify patients with painful $(P)$ or painless (NoP) diabetic polyneuropathy (DPN). Diagnostic accuracy of StEPS is compared to that of the full 47-item interview and 39-item physical examination. Numbers denote areas under the curves (AUCs). 
Table 1

Demographic and clinical characteristics of the study participants.

\begin{tabular}{|c|c|c|c|c|c|}
\hline & \multicolumn{2}{|l|}{ DPN } & \multirow[t]{2}{*}{ DM2 w/o DPN } & \multirow[t]{2}{*}{$\mathrm{HC}$} & \multirow[t]{2}{*}{$P$} \\
\hline & $\mathbf{P}$ & NoP & & & \\
\hline $\mathrm{N}$ & 61 & 19 & 25 & 20 & \\
\hline Age, median $Y(Q 1, Q 3)$ & $54(49,59)$ & $57(50,63)$ & $55(50,59)$ & $61(56,66)$ & $<0.05^{\star}$ \\
\hline Women, N (\%) & $25(41)$ & $4(21)$ & $6(24)$ & $7(35)$ & $<0.01$ \\
\hline Duration of diabetes, median $Y(Q 1, Q 3)$ & $10(6,13)$ & $15(5,20)$ & $5(4,13)$ & & $<0.05^{\star}$ \\
\hline HbA1c, median \% (Q1, Q3) & $7.3(6.4,8.4)$ & $7.6(7.1,8.1)$ & $7.5(6.3,8.9)$ & & NS \\
\hline DNS score, median (Q1, Q3) & $3(3,4)$ & $2(1.5,2)$ & $0(0,0)$ & $0(0,0)$ & $<0.001^{*}$ \\
\hline \multicolumn{6}{|l|}{ Toronto CNS scores } \\
\hline Symptoms, median (Q1, Q3) & $3(3,5)$ & $2(1.5,2)$ & $0(0,0)$ & $0(0,0)$ & $<0.001^{*}$ \\
\hline Sensory tests, median (Q1, Q3) & $3(2,4)$ & $2(1.5,4)$ & $1(0,1)$ & $1(0,1)$ & $<0.001^{*}$ \\
\hline Duration of pain, median $Y(Q 1, Q 3)$ & $4(2,7)$ & & & & \\
\hline Pain intensity (NRS), median (Q1, Q3) ${ }^{\dagger}$ & $7(5.0,8.0)$ & & & & \\
\hline Family history of diabetes, $\mathrm{N}(\%)$ & $48(79)$ & $17(89)$ & $23(92)$ & $7(35)$ & $<0.001$ \\
\hline Family history of neuropathy, N (\%) & $32(52)$ & $5(26)$ & $4(16)$ & $0(0)$ & $<0.001$ \\
\hline
\end{tabular}

* ANOVA followed by Tukey's test for multiple comparisons revealed significant differences in the age of patients with DPN-P and HC subjects, and in the diabetes duration of patients with DPN-NoP and DM2 w/o DPN. DNS and Toronto CNS symptom scores were significantly different between all subgroups of patients with DM2, Toronto CNS sensory tests scores between DPN-P or DPN-NoP and DM2 w/o DPN.

$\dagger$ Global pain intensity in the week prior to enrollment.

CNS, Clinical Neuropathy Scoring System; DM2, type 2 diabetes mellitus; DNS, Diabetic Neuropathy Symptom score; DPN, diabetic polyneuropathy; HbA1c, hemoglobin A1c; Hx, history; NoP, no pain; NRS, numerical rating scale from 0 to 10; NS, not significant; P, pain; Q1 and Q3, first and third quartiles; $Y$, years. 


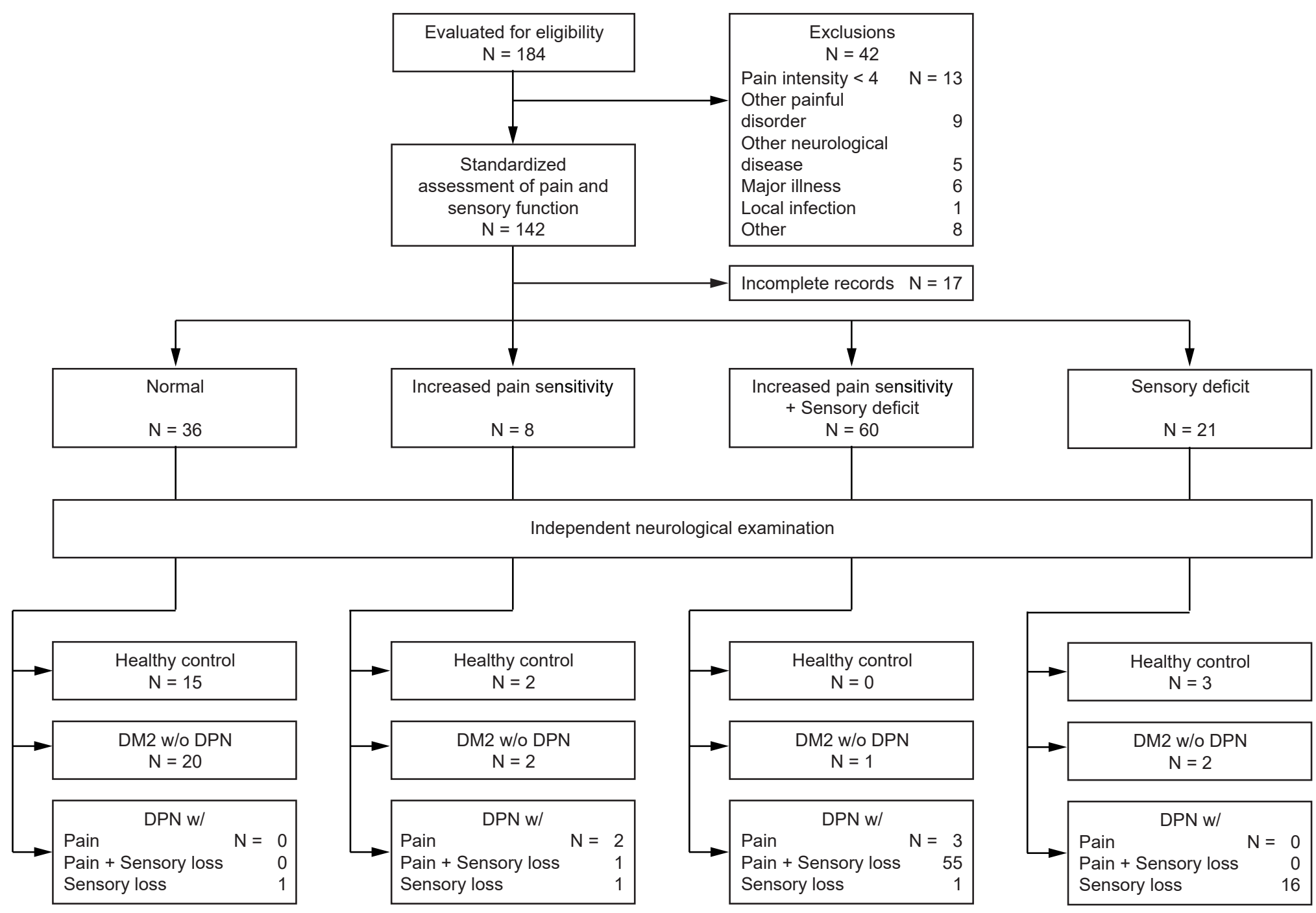




\section{DPN-P}

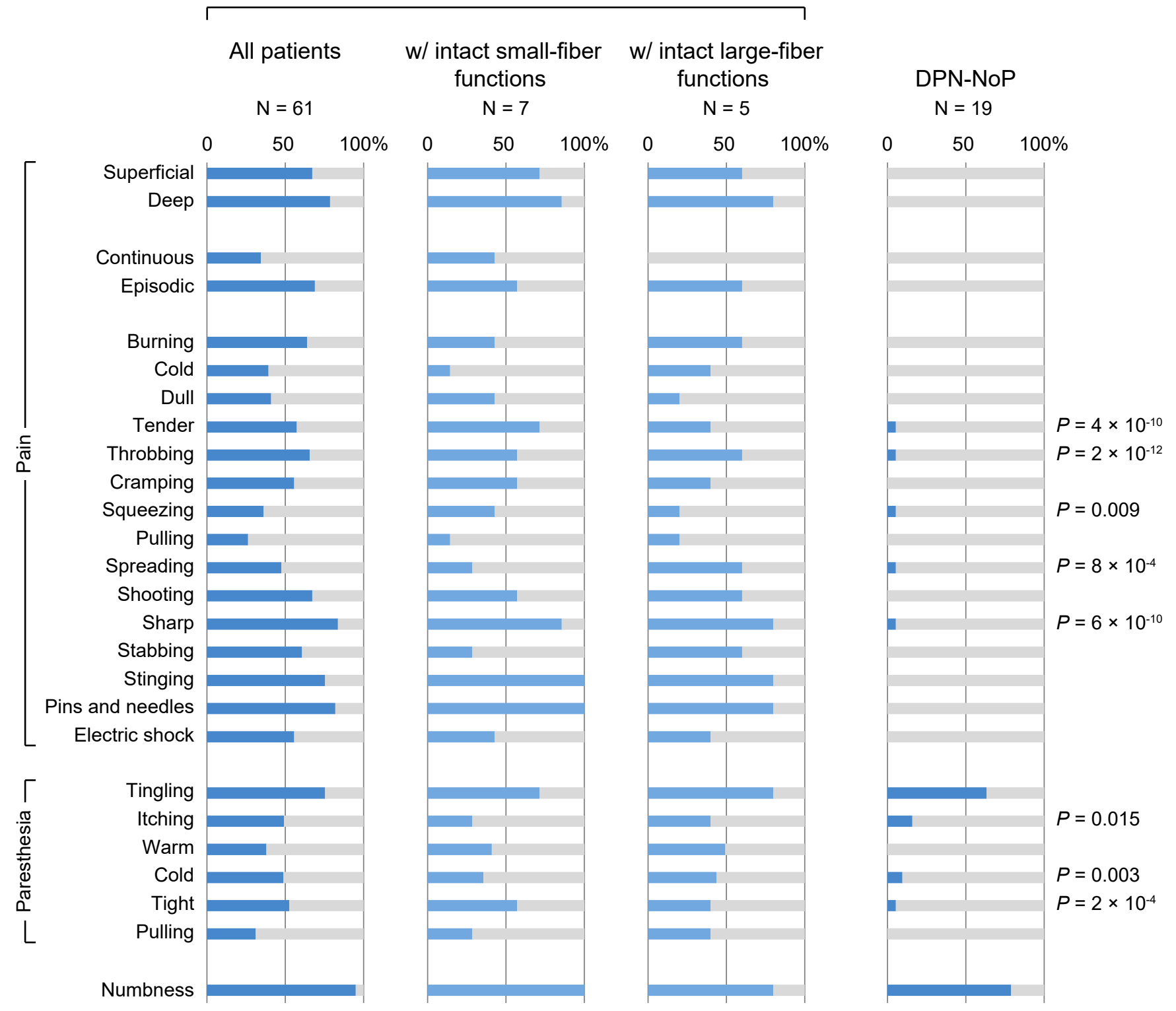




\section{DPN-P}

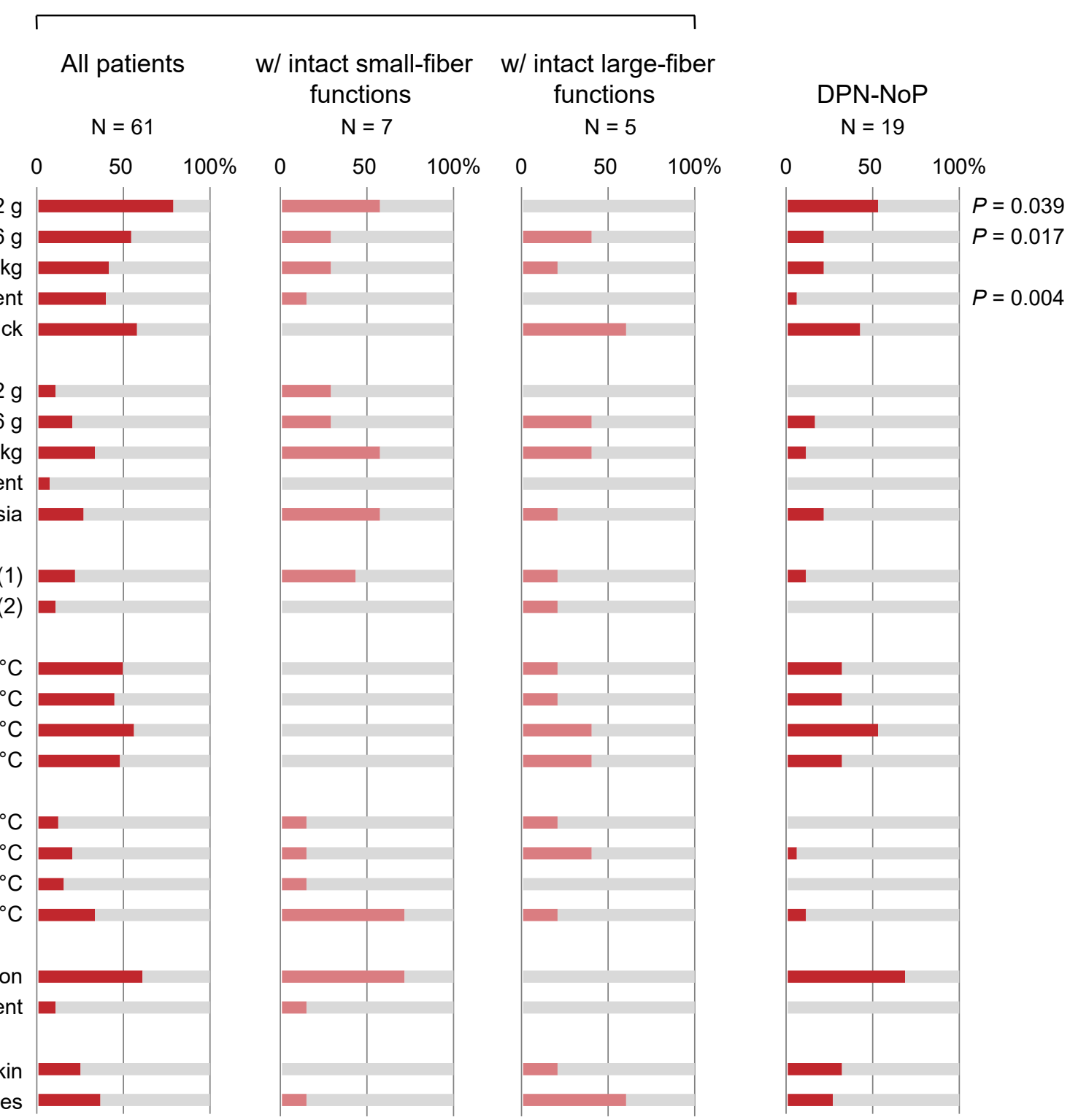


A Numbness

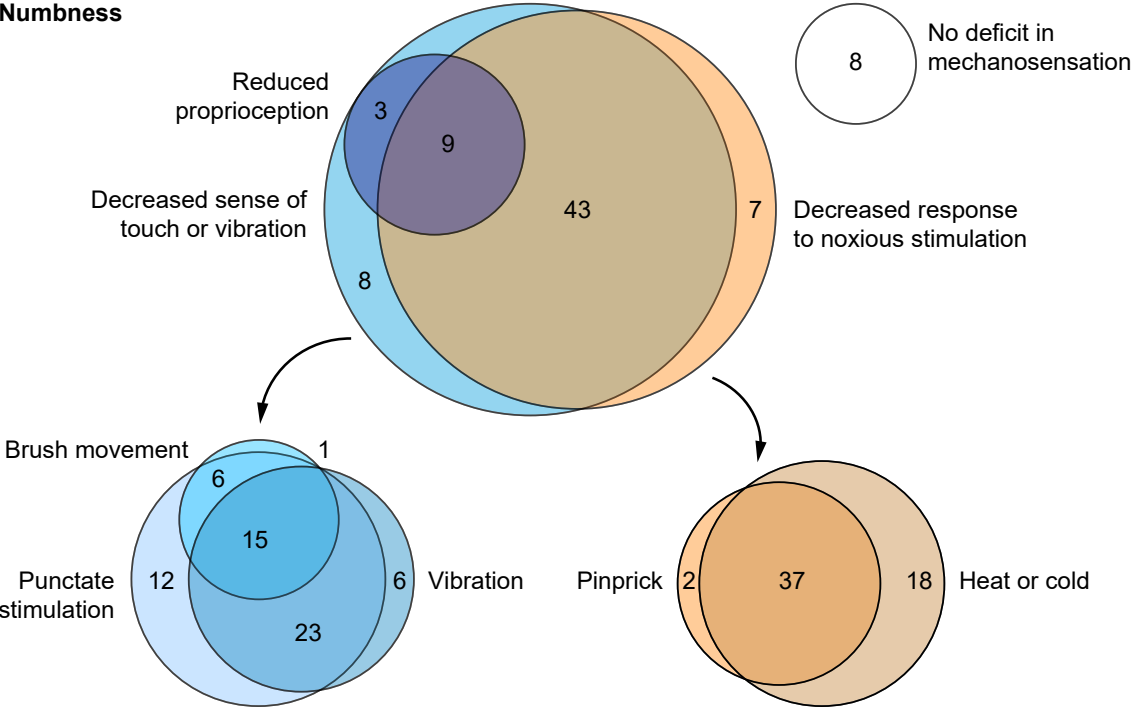

B

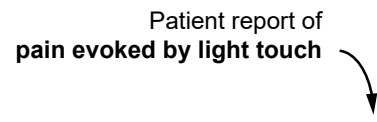

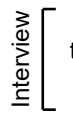

Evoked pain ends with the removal of the stimulus

$\underline{ \pm}[\quad$... outlasts the stimulus

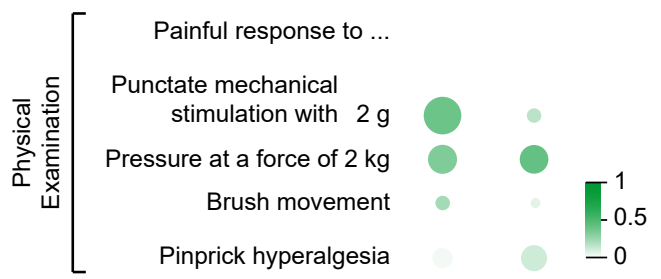

Patient report of

increased sensitivity to pressure

C

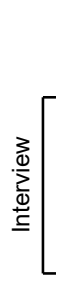

Patient report of cold-evoked pain )

warmth-evoked pain

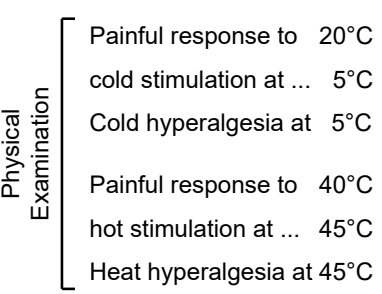


A

\begin{tabular}{|c|c|c|c|c|c|c|c|c|}
\hline \multirow{3}{*}{$\begin{array}{r}\text { Mechanical } \\
\text { stimuli }\end{array}$} & \multicolumn{4}{|c|}{$\begin{array}{l}\text { Decreased } \\
\text { - response - }\end{array}$} & \multicolumn{4}{|c|}{ Painful } \\
\hline & $\begin{array}{ll}\text { D) } & \text { O) } \\
\sim & \stackrel{d}{N}\end{array}$ & $\stackrel{\text { D }}{\sim}$ & & & $\stackrel{\sigma}{N}$ & & $\frac{\mathbb{P}}{N}$ & \\
\hline & 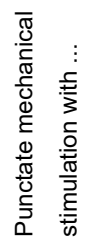 & 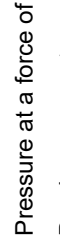 & 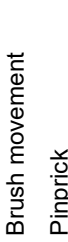 & $\begin{array}{l}\text { 은 } \\
\stackrel{0}{0} \\
\stackrel{0}{2}\end{array}$ & 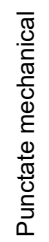 & 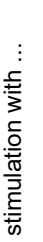 & 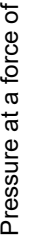 & 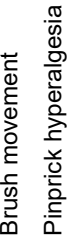 \\
\hline
\end{tabular}
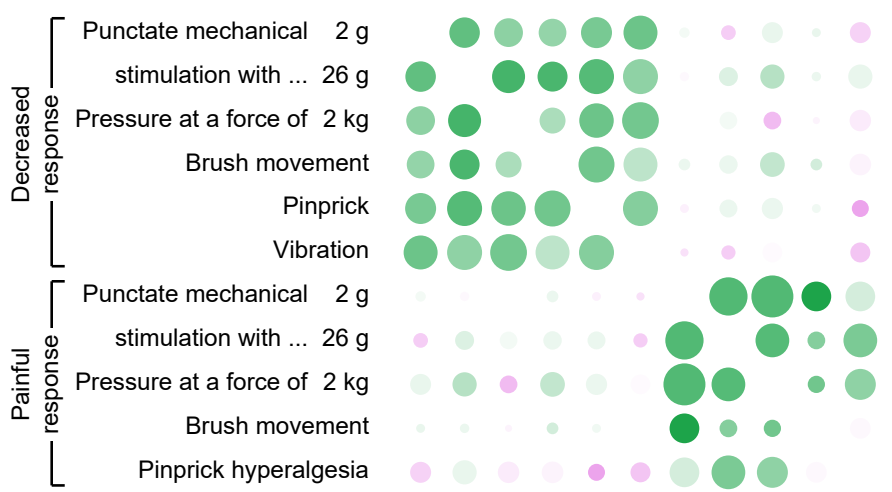

Punctate mechanical $2 \mathrm{~g}$

Pressure at a force of $2 \mathrm{~kg}$

Brush movement

Pinprick hyperalgesia
B

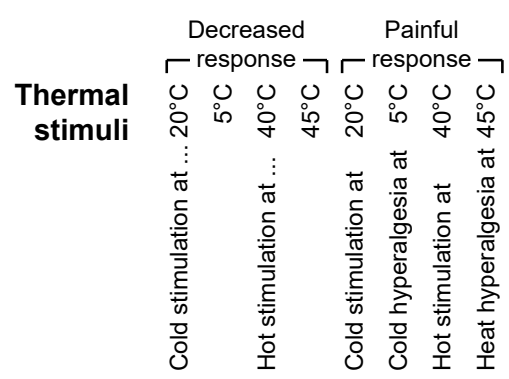

C Cold stimulation at $20^{\circ} \mathrm{C}$

$55^{\circ} \mathrm{C}$

¿ $\frac{1}{2}$ Hot stimulation at $40^{\circ} \mathrm{C}$

$\triangle$

L Cold stimulation

$45^{\circ} \mathrm{C}$
$20^{\circ} \mathrm{C}$

$\bar{\infty}$ C Cold hyperalgesia at $5^{\circ} \mathrm{C}$

त :

$\left\llcorner\right.$ Heat hyperalgesia at $45^{\circ} \mathrm{C}$
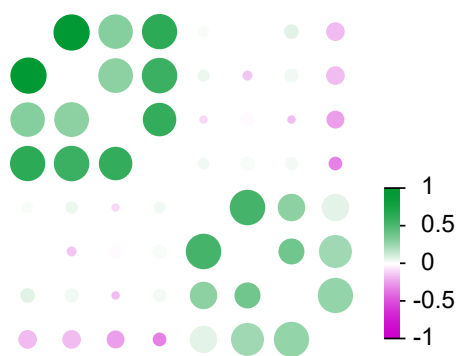
B

Component 1 (16\%)
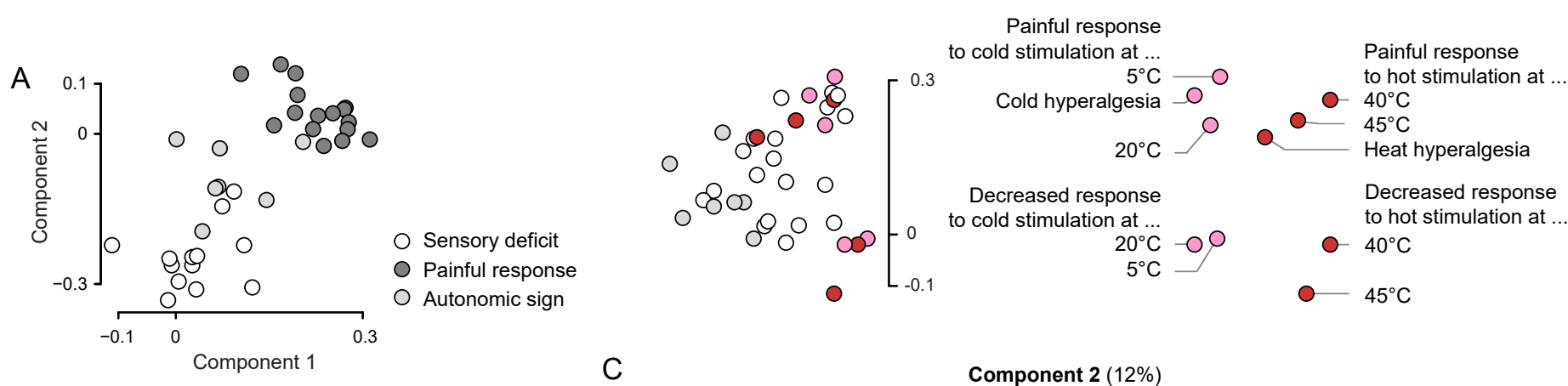

C

Component 2 (12\%)

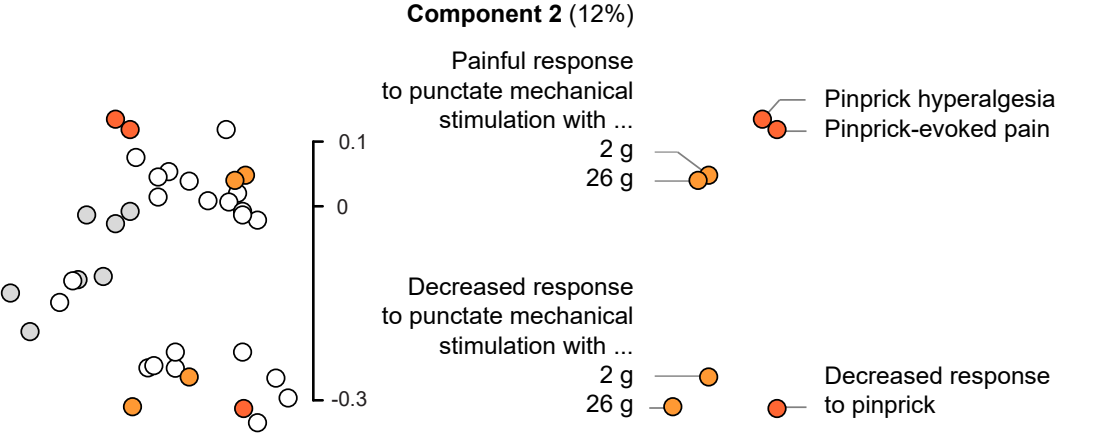




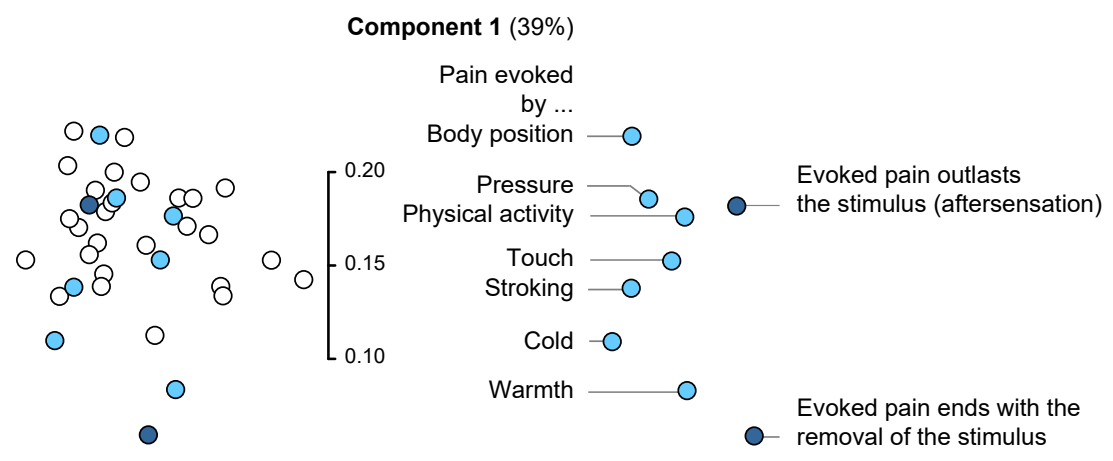


Distinction between ...

DM2 w/o DPN and DPN-P

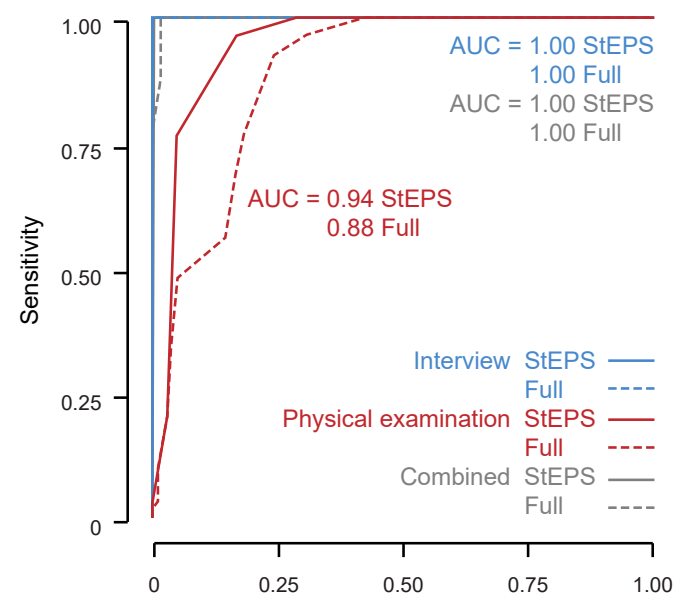

DM2 w/o DPN and DPN-NoP

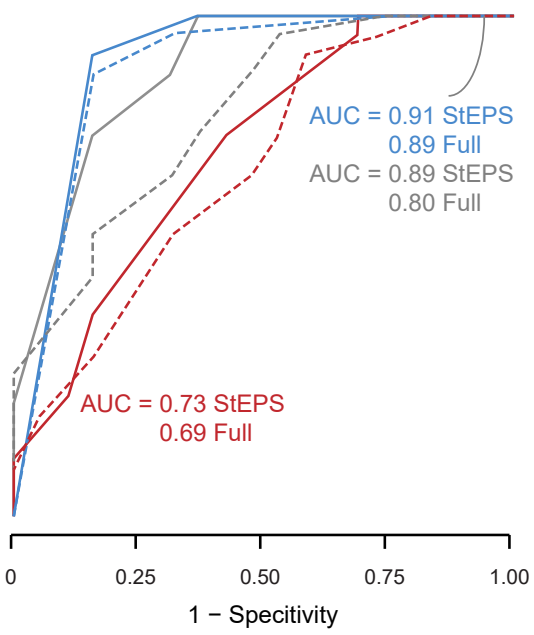

DPN-P and DPN-NoP

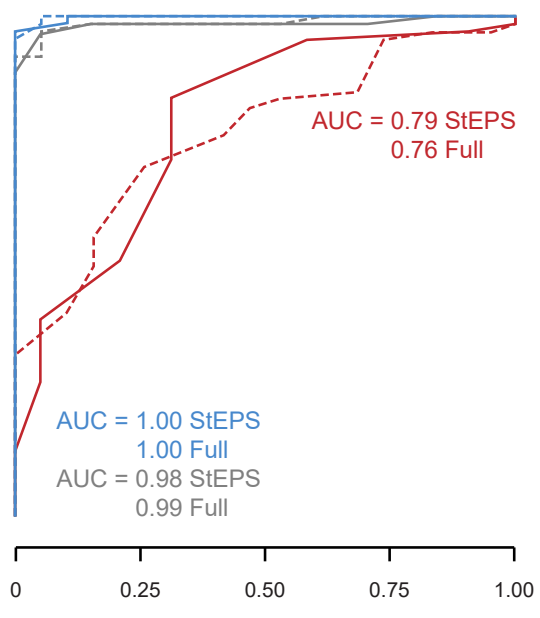




\section{Supplemental Table 1}

Area under the ROC curves for individual StEPS items in the distinction between ...

\section{DM2 w/o DPN and DPN-P}

\begin{tabular}{|c|c|c|c|}
\hline Item & AUC & Item & AUC \\
\hline Interview & & Interview & \\
\hline Numbness & $0.95(0.91,1.00)$ & Numbness & $0.87(0.77,0.98)$ \\
\hline Deep pain & $0.90(0.85,0.95)$ & Tingling paresthesia & $0.82(0.70,0.93)$ \\
\hline Stinging pain & $0.88(0.83,0.94)$ & Physical examination & \\
\hline Tingling paresthesia & $0.88(0.83,0.94)$ & Reduced sense of vibration & $0.78(0.66,0.91)$ \\
\hline Superficial pain & $0.85(0.79,0.91)$ & Decreased response to pinprick & $0.71(0.60,0.82)$ \\
\hline Episodic pain & $0.85(0.79,0.91)$ & & \\
\hline $\begin{array}{l}\text { Evoked pain outlasts the } \\
\text { stimulus (aftersensation) }\end{array}$ & $0.83(0.77,0.89)$ & & \\
\hline Burning pain & $0.83(0.77,0.89)$ & & \\
\hline Pain caused by pressure & $0.79(0.72,0.85)$ & & \\
\hline Cramping pain & $0.78(0.72,0.84)$ & & \\
\hline Cold paresthesia & $0.75(0.69,0.82)$ & & \\
\hline Itching paresthesia & $0.73(0.66,0.81)$ & & \\
\hline Pain triggered by touch & $0.72(0.66,0.78)$ & & \\
\hline Pain provoked by stroking & $0.71(0.65,0.78)$ & & \\
\hline Cold pain & $0.70(0.64,0.77)$ & & \\
\hline \multicolumn{4}{|l|}{ Physical examination } \\
\hline Decreased response to pinprick & $0.80(0.73,0.86)$ & & \\
\hline $\begin{array}{l}\text { Decreased response to punctate } \\
\text { mechanical stimulation with } 2 \mathrm{~g}\end{array}$ & $0.78(0.68,0.88)$ & & \\
\hline Reduced sense of vibration & $0.75(0.65,0.84)$ & & \\
\hline
\end{tabular}

\section{DM2 w/o DPN and DPN-NoP}

\section{.}




\section{Supplemental Table 2}

Continued

\section{DPN-P and DPN-NoP}

\begin{tabular}{ll}
\hline Item & AUC \\
\hline Interview & \\
Deep pain & $0.90(0.85,0.95)$ \\
Stinging pain & $0.88(0.83,0.94)$ \\
Superficial pain & $0.85(0.79,0.91)$ \\
Episodic pain & $0.85(0.79,0.91)$ \\
Burning pain & $0.83(0.77,0.89)$ \\
Pain caused by pressure & $0.79(0.72,0.85)$ \\
Cramping pain & $0.78(0.72,0.84)$ \\
Evoked pain outlasts the & $0.78(0.68,0.87)$ \\
stimulus (aftersensation) & \\
Pain provoked by stroking & $0.71(0.65,0.78)$ \\
Cold pain & $0.70(0.64,0.77)$ \\
Cold paresthesia & $0.70(0.61,0.80)$
\end{tabular}




\section{Supplemental Table 2}

Sensitivity and specificity to identify DPN-P

\begin{tabular}{|c|c|c|c|c|}
\hline & \multicolumn{2}{|c|}{$\begin{array}{l}\text { Standardized evaluation of pain } \\
\text { and somatosensory function }\end{array}$} & \multicolumn{2}{|l|}{ DN4 } \\
\hline & Full version & StEPS & 10 Items & 7 Items \\
\hline Sensitivity, \% & $100(94,100)$ & $100(94,100)$ & $85(74,93)$ & $90(80,96)$ \\
\hline Specificity, \% & $89(79,95)$ & $98(92,100)$ & $98(92,100)$ & $95(87,99)$ \\
\hline PPV, \% & $90(80,96)$ & $98(91,100)$ & $98(90,100)$ & $95(86,99)$ \\
\hline NPV, \% & $100(94,100)$ & $100(94,100)$ & $88(78,94)$ & $91(82,97)$ \\
\hline
\end{tabular}

Values in brackets denote the $95 \%$ confidence interval.

DN4, Douleur Neuropathique en 4 Questions [1]; DPN-P, painful diabetic polyneuropathy; NPV, negative predictive value; P, pain; PPV, positive predictive value.

\section{References}

[1] Bouhassira D, Attal N, Alchaar H, Boureau F, Brochet B, Bruxelle J, Cunin G, Fermanian J, Ginies P, GrunOverdyking A, Jafari-Schluep H, Lanteri-Minet M, Laurent B, Mick G, Serrie A, Valade D, Vicaut E. Comparison of pain syndromes associated with nervous or somatic lesions and development of a new neuropathic pain diagnostic questionnaire (DN4). Pain 2005;114(1-2):29-36. 